\title{
Guía documental sobre roles sexuales: masculinidad y feminidad
}

\author{
Julia Sebastián \\ Valentina del Valle \\ Carlos Martínez \\ Emilio González \\ Francisco J. Fernández \\ Bernardo Moreno
}

\section{INTRODUCCION}

El presente artículo tiene como finalidad recopilar y ofrecer al lector interesado una panorámica lo más actualizada posible —si bien no lo más exhaustiva posible- sobre el tema de los roles sexuales. Nuestro trabajo se sitúa en la perspectiva más actual de tratamiento de este área de estudio, que gira fundamentalmente alrededor de los constructos de masculinidad, feminidad y androginia.

Se hace, pues, necesario señalar, en primer lugar, esta restricción impuesta a esta guía documental desde consideraciones de tipo tanto temático como metodológico. El tema de los roles sexuales está fuertemente vinculado a otros muchos como el de la identidad sexual, el proceso de tipificación sexual, el papel que puede jugar la familia en este proceso, las diferencias sexuales, etc. Incluso, el estudio de los roles sexuales es susceptible de hacerse desde diferentes enfoques: antropológico, sociológico, psicológico, histórico-cultural, económico, sexológico, etc. Por todo ello, se hacía necesario introducir una serie de linderos que demarcasen más concretamente el área a cubrir. En este sentido, nuestro acercamiento al tema se ha realizado desde la óptica de la moderna concepción de la masculinidad y feminidad, dentro de lo que se ha venido en llamar «los teóricos de la androginia».

Etimológicamente la palabra «androginia» proviene del griego «andro» hombre, y «ginia» mujer; precisamente con este término se da a entender que el sujeto andrógino asume y exterioriza el significado social de ambas polaridades.

El arquetipo andrógino ha existido en casi todas las culturas, bien a través de mitos o leyendas, filosofías o religiones ${ }^{1}$. Sin embargo, su operativización a nivel psicológico (cuantificable) no fue realizada hasta 1974, año en que Sandra Bem dio a conocer su escala de medida del rol sexual (BSRI), y la forma de clasificar a los sujetos como masculinos, femeninos indiferenciados y andróginos ${ }^{2}$.

Más concretamente, la androginia es la integración de atributos definidos culturalmente como masculinos y femeninos o, si se prefiere, el equilibrio entre lo instrumental y lo expresivo o lo «agency» y «communion», etc. Se sitúa en el área del género y no del sexo, por lo que no debe de ser confundida con ningún tipo de elección de objeto sexual (homosexualidad o bisexuali- 
dad), o con otras series de cuestiones como la falta de identificación sexual, hermafroditismo, etc.

El tema de la masculinidad y feminidad ha ido poco a poco evolucionando de acuerdo a las coordenadas sociales en las que se inscribía. De los supuestos subyacentes al modelo clásico de la masculinidad y feminidad (unidimensionalidad, bipolaridad, estrecha relación con el sexo biológico, etc.) se ha pasado a los presupuestos del modelo actual (bidimensionalidad, ortogonalidad, laxa relación con el sexo, etc.), sin que por ello se haya todavía establecido el «verdadero» modelo teórico que asuma todas las implicaciones reales del fenómeno. No obstante, la investigación actual ya está dirigiendo sus miras hacia otros horizontes, a partir precisamente de los resultados más recientes.

El estudio de la masculinidad y feminidad, cualquiera que sea su perspectiva, es un tema tan complejo y rico en implicaciones que ha dejado de tener una ubicación dentro de una disciplina psicológica concreta. Si tradicionalmente era tratado en los manuales de Psicología Diferencial, más concretamente - y algo erróneamente- en el capítulo de las diferencias sexuales, actualmente puede y debe ser tratado en disciplinas tales como la Psicología de la Personalidad, la Psicología Evolutiva, la Psicología Social, o la Psicología Cognitiva (a partir de su estudio a través de esquemas cognitivos). En cualquiera de ellas lo más importante a resaltar es que la relación directa que existía anteriormente entre sexo y género, hoy por hoy, resulta dudosa.

En este terreno del sexo y del género existen una serie de obras que se consideran clásicas. A continuación expondremos las más importantes:

BAKAN, D. The duality of buman existence, Chicago: Rand McNally, 1966.

BILLER, H. B. Father, child, and sex role, Lexington, Mass: Heath Lexington Books, 1971.

ELLIS, H. Man and Woman: A study of human secondary sex characteristics, Nueva York: Scribner, 1904.

GrambS, J. D., y WAETjEN, W. B. Sex does it make a difference? Belmont, California: Buxbury, 1975.

HEILBRUM, C. Toward a recognition of androgyny, Nueva York: Knopf, 1973.

MEAD, J. Male and Female, Nueva York, Morrow, 1949.

MONEY, J. (ed.). Sex research: New developments, Nueva York, Rinehart and Winston, 1965.

OAKLEY, A. Sex, gender and society, Nueva York, Harper and Row, 1972.

PARSONS, T., y BALES, R. F. Family, socialization and interaction process, Nueva York: Free Press of Glencoe, 1955.

STOLLER, R. J. Sex and gender, Nueva York: Science House, 1968.

STOLLER, R. J. Sex and gender: the development of masculinity and feminity, vol. I, Nueva York: J. A ronson, 1974.

StOLLER, R. J. Sex and gender, vol. 2: The transexual experiment, Nueva York: Aronson, 1975.

Terman, L. M., y MiLes, C. S. Sex and personality, Nueva York: Mc Graw-Hill, 1936.

TYLER, L. The psychology of buman differences, Nueva York: Appleton-CenturyCrofts, 1965.

Yorburg, B. Sexual identity: sex roles and social change, Nueva York: Wiley, 1974

A continuación, y como muestra de lo que exponíamos anteriormente sobre la variedad y amplitud del tratamiento del tema de los roles sexuales, ofrecemos primeramente una lista de libros más o menos actuales que pueden ayudar a completar el tema. Posteriormente, en este mismo sentido, señalaremos algunos otros que pueden despertar la curiosidad del lector.

ADAmS, C., y LaURIKIETIS, R. The gender trap: A closer look at Sex Roles, vol. 1, Education and Work-sellers, Jill (ed.), 1977.

ADAMS, C., y LAURIKIETIS, R. The gender trap: A closer look at Sex Roles, vol. 2, Sex and Marriage, 1977.

ADAMS, C., y LAURIKIETIS, R. The gender trap: A closer look at Sex Roles, vol. 3, Message and Images, 1977. 
ALLGEIER, E., y MCCORMICK, N. Invisible boundaries: Gender roles and sexual Bebavior, Londres: Mayfield Pub., 1982.

ICKES, W., y KNOWLES, E. S. (eds.). Personality, Roles and Social Behavior, Nueva York: Springer-Verlag, $1982^{3}$.

MOBERLY, E. R. Psychogenesis: The early development of gender identity, Londres: Toutledge and Kegan Paul, $1983^{4}$.

MONEY, J., y TUCKER, P. Sexual signature, Boston: Little Brown, 1975.

ORASANU, J., y SLATER, M. K., y A DLER, L. L. (eds.) «Language, sex and gender: Does "The Difference" make a difference?» Annals of the New York Academy of Sciences, vol. 327, Nueva York: New York Academy of Sciences, 1979's.

RICHARDSON, L. W. The dynamics of sex and gender: $A$ sociological perspective, Boston: Houghton Mifflin, 1981.

BUTLER, P. Self-assertion for women: a guide to becoming androgyneus, San Francisco: Canfield Press, 1976.

COLEGRAVE, S. The spint of the valley: androgyny and Chinese Thought, Londres: Vivago, 1979.

Jung, C. G. Aspects of the Feminine, Princenton: University Press, 1982.

LIBIS, J. Le mytbe de l'andogyne, París: Berg International, 1980.

PRINGle, M. B., y STERICKER, A. (eds.). Sex role in literature, Nueva York: Logman, 1980.

SARgENT, A. G. The androgynous manager, Nueva York: Amacom, 1981.

\section{ORGANIZACION DE LA GUIA}

Empezaremos indicando las obras editadas en castellano. Posteriormente, como es costumbre en este tipo de trabajos, se hará referencia en primer lugar, a las fuentes primarias o directas según el siguiente orden:

1. Tesauro. 2. Tratados, textos y monografías. 3. Revistas. 4. Compilaciones. 5. Actas, series y guías.

Las fuentes secundarias que se mencionarán serán:

6. Revisiones. 7. Revistas de resúmenes. 8. Catálogos e índices.

Como anteriormente se ha mencionado, deliberadamente hemos obviado obras que tratasen el tema desde otra perspectiva que no fuera la psicología, e incluso, dentro de ella, se ha tenido que renunciar - en aras de la concreción, operatividad y clarificación - a temas afines. Nos gustaría señalar aquí que el proceso de delimitación y toma de decisiones del área a tratar en la presente guía no ha sido una tarea fácil, ya que existe una cantidad ingente de bibliografía sobre el tema de los roles sexuales. Específicamente, hemos intentado alejarnos incluso de obras específicas sobre la psicología femenina o masculina, temas de la mujer desde una óptica psicosociológica o el tema de los estereotipos (que por sí mismos constituyen la elaboración de otra guía); asimismo, hemos huido de cuestiones excesivamente concretas.

Por todo ello es necesario advertir al lector que éste no pretende ser un trabajo bibliométrico. Los autores se reservan cierto grado de arbitrariedad en función de sus preferencias y criterios.

\section{OBRAS EN CASTELLANO}

En este apartado mencionaremos inicialmente los libros y artículos en castellano; hemos intentado separar aquellos que puedan carecer de interés específico ${ }^{6}$. Posteriormente, reseñaremos los trabajos de investigación más importantes, algunos de los cuales provienen de memorias de licenciatura o de tesis doctorales. 
Barberá Heredia, E. Psicología de la mujer y roles sexuales. Psicológica, 1983, 4, 49-65.

BEAUVOIR DE, S. El segundo sexo, vols. I y II, Buenos Aires: Siglo XX, 1975.

FERNANDEZ SÁNCHEZ, J. Nuevas perspectivas en la medida de la masculinidad y feminidad. Tesis doctoral. Madrid: Ediciones de la Universidad Complutense, 1983.

GIANINI BELloti, E. A favor de las niñas: la influencia de los condicionamientos sociales en la formación del rol femenino en los primeros años de vida, Caracas, Venezuela: Monte Avila Editores, 1980 (original de 1973).

MARCET CABRAL, $C$. «Nuevas aportaciones al estudio sobre el rol sexual: la teoría del esquema cognitivo del sexo". Quaderns de Psicologia, 1983, 7, 41-53.

MARTINEZ BENLlOCH, I. Aportaciones a la medida de los constructores de género (Lecturas básicas), Valencia: Facultad de Psicología, Universitat de Valencia, 1986.

MONEY, J., y EHRHARDT, A. Desarrollo de la sexualidad bumana, diferenciación y dimorfismo de la identidad de género, Madrid: Morata, 1982 (edición original de 1972)

MOYa MORALES, M. «Identidad, roles y estereotipos de género». Revista de Psicología General y Aplicada, 1985, 403, 457-472.

Rocheblave-SPENLE, A. M. Lo masculino y lo femenino en la sociedad contemporánea, Madrid: Ciencia Nueva, 1968 (original de 1966).

\section{Trabajos de investigación ${ }^{7}$}

Aguiñiga Benito, C. Androginia y ajuste de pareja. Memoria de licenciatura, Universidad Autónoma de Madrid, 1986.

FERNÁNDEZ SÁNCHEZ, J. Nuevas perspectivas en la medida de la masculinidad y feminidad. Tesis doctoral, Universidad Complutense de Madrid, 1982.

Moreno, B.; AgufÑIGA, C., y SEBASTIÁN, J. «¿Es transaccional la androginia?, en Sexualidad en un mundo en cambio. I Congreso Español de Sexología. Fundación Banco Exterior, Madrid, 1985, págs. 416-433.

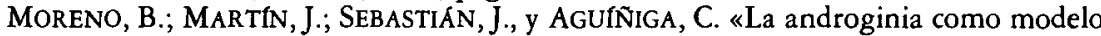
de salud mental: un estudio de la eyaculación precoz», en Sexualidad en un mundo en cambio. I Congreso Español de Sexología. Fundación Banco Exterior, Madrid, 1985, págs. 436-453.

MOYa MORALES, M. «Aproximación psicosocial a los roles sexuales». Memoria de licenciatura. Universidad de Granada, 1984.

QUiRoGA, M. A.; SÁNCHEZ, M. F.; CHACON, F., y ForTEZA, J. A. «Relaciones entre la autoestima, masculinidad y feminidad y la autoevaluación bilingüe». Informes de Psicologia, 1985, 17, 125-143.

SEBASTIÁN HERRANZ, J. La androginia como indice de flexibilidad comportamental. Tesis doctoral. Universidad Autónoma de Madrid, 1986.

\section{TESAURO}

El Tesauro ayuda al investigador a establecer relaciones semánticas, funcionales y sintácticas entre diversos términos relacionados ${ }^{8}$. En nuestro caso el descriptor Roles Sexuales estaba incluido bajo el término genérico de Roles ${ }^{9}$, y tiene un mismo nivel de amplitud que los términos Androgyny, Masculinity, Feminity y Sex Role Attitudes.

A partir de esta información hemos tratado de construir un mapa de relaciones entre los distintos descriptores (Tabla 1).

\section{TRATADOS, TEXTOS Y MONOGRAFIAS}

Como se ha informado anteriormente, los criterios de selección de las obras reseñadas a continuación responden a diversas consideraciones y han estado sujetos a las directrices, formación y prioridades marcadas por los propios autores que la han realizado. 


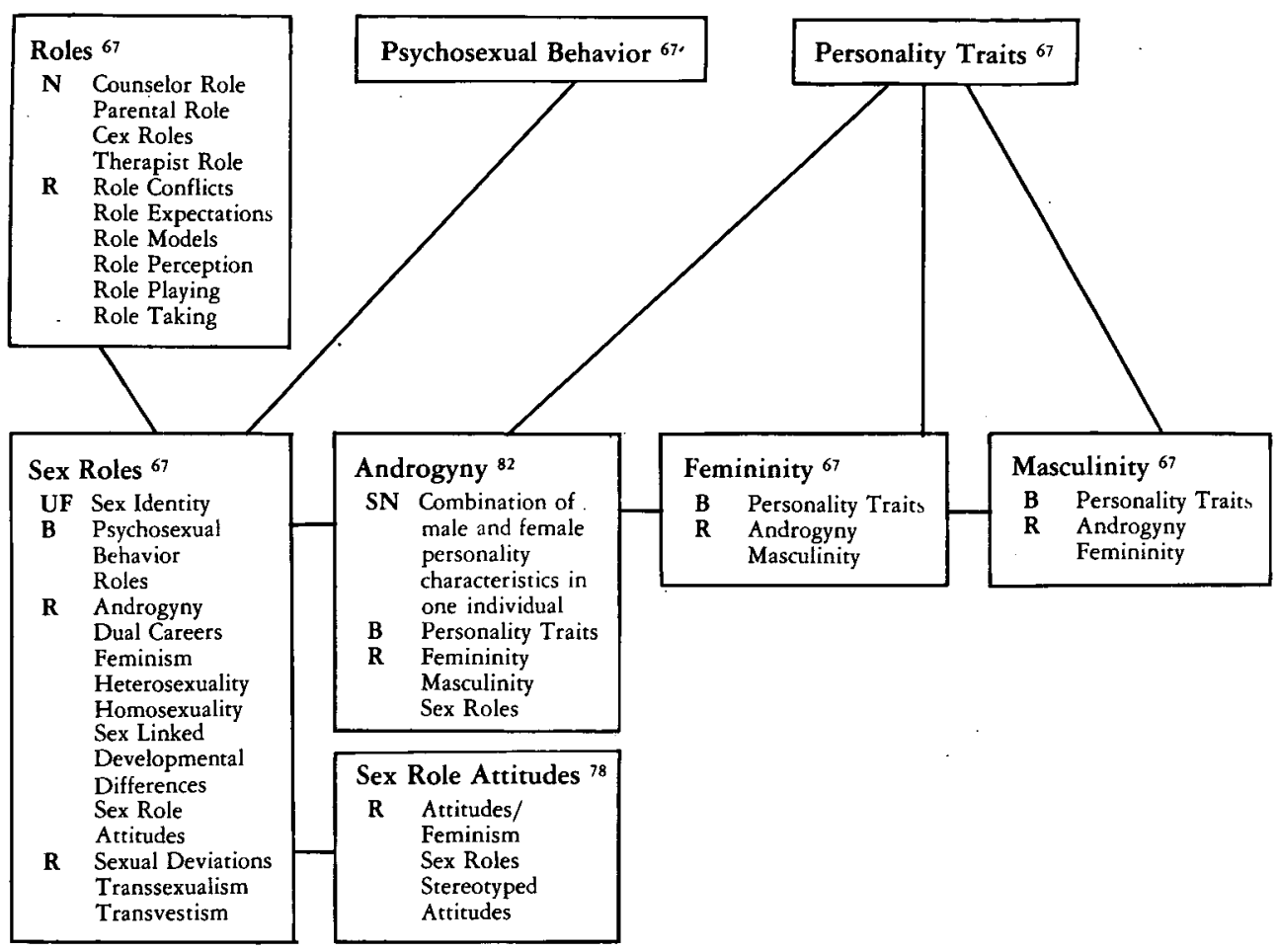

Cuando la obra es reseñada en el Contemporary Psychology, su referencia figura en las notas al final del artículo.

BASOW, S. A. Sex-role stereotypes: Traditions and alternatives, Monterrey, California: Brooks-Cole, $1980^{10}$.

BERNICE, L. Becoming a woman: The socialization of gender, Springfield, Ill.: Charles C. Thomas, $1981^{11}$.

CHAFETZ, J. S. Masculine, Femenine or Human? An overviwe of the sociology of the gender roles, 2.a ed., Itaca, Ill.: Peacook, $1978^{12}$.

CoOK, E. P. Psychological Androgyny, Nueva York: Pergamon Press, $1985^{13}$.

DEAUX, K. The behavior of women an men, Monterrey, California: Brooks-Cole, 1976.

DUBBerT, J. L. A man's place: masculinity in transition, Englewood Cliffs, Nueva Jersey: Prentice-Hall, 1978.

FrankLIN, C. W. The changing definition of Masculinity, Nueva York: Plenum Press, 1984.

FORISHA, B. L. Sex roles and personal awareness, Morristown, Nueva Jersey: General Learning Press, $1978^{14}$.

Heilbrum, A.B., JR. Human Sex-Role Behavior, Nueva York: Pergamon Press, $19811^{15}$.

KAPLAN, A. G., y SEDneY, M. A. Psychology and sex roles: an androgynous perspective, Boston: Little Brown, 1980.

Katchadourian, H. A. Human sexuality. A comparative and developmental perspective, Berkeley: University of California Press, 1981.

LATORRE, R. A. Sexual Identity: Implications for Mental Health, Chicago: NelsonHall, $1979^{16}$.

OLDS, L. E. Fully buman: bow everyone can integrate the benefits of masculine and feminine sex roles, Englewood Cliffs, Nueva Jersey: Prentice-Hall, 1981.

PARSONS, J. E. The psychology of sex differences and sex roles, Nueva York: McGrawHill, 1980.

PLECK, J. H. The myth of masculinity, Cambridge Mass.: MIT Press, $1981^{17}$.

RosenBERG, B. G., y BUTTON-SMITH, B. Sex and identity, Nueva York: Holt Rinehart and Wiston, $1972^{18}$.

RossI, A. S. Gender and the life course, Nueva York: Aldine, 1985. 
Sargent, A. G. Beyond sex roles, St. Paul: West Pub. Co., 1977.

SCHAFFER, K. F. Sex Roles and Human Behavior, Cambridge, Mass.: Winthrop, $19811^{19}$

SingER, J. "Androgyny: Toward a New Theory of Sexwality», Garden City, Nueva York: Anchor Press, Doubleday, $1976^{20}$.

SPENCE, T. J., y HELMREICH, R. L. Masculinity and Feminity: Their psychological dimensions, correlates, and Antecedents, Austin: University of Texas Press, $1978^{21}$.

STOCKARD, J.; JoHNSON, M. N. Sex roles, sex inequality and sex role development, Prentice Hall, 1980.

Unger, R. K. Female and Male. Psychological Perspectives, Nueva York: Harper and Row, $1979^{22}$

WeITz, S. Sex Roles: Biological, Psychological and Social Foundations, Nueva York: Oxford University Press, 1977.

WESLEY, F., y WESLEY, C. Sex roles psychology, Nueva York: Human Sciences Press, $1977^{23}$.

WILliams, J. E., y BeST, D. L. Measuring sex stereotypes, Londres: Sage Publications, 1982.

Zolla, E. The Androgyne: The creative tension of male and femule, Crossroad, Nueva York, 1981

\section{REVISTAS}

La revista que actualmente recoge un mayor número de artículos relacionados con el tema es Sex Roles (Sex Roles: A Journal of Research). Esta revista se empezó a publicar en 1975 bajo la dirección de P. A. Katz y su periodicidad es mensual (editada por Plenum Press, 233 Spring St., Nueva York, NY 10013). Como su nombre indica, esta publicación es la más específica con respecto al tema que nos ocupa.

Otras tres revistas pueden ser consideradas como relevantes en cuanto al número de artículos publicados sobre el tema de la masculinidad y feminidad aunque su contenido es mucho más amplio:

Psychological Reports. Esta revista recoge fundamentalmente artículos que hacen referencia a cuestiones metodológicas y de análisis estadístico en lo que se refiere al área de los roles sexuales (editada bimensualmente desde 1955; dirigida por C. H. Ammons y R. B. Ammons, Box 9229, Mossoula, MT 59807).

Journal of Personality and Social Psychology. Es una de las revistas más importantes en el vasto campo del estudio de la personalidad y sus implicaciones sociales (editada mensualmente desde 1965 por American Psychological Association, 1200 17th St. N. W.; Washington DC 20036).

Journal of Consulting and Clinical Psychology. Aparecen artículos relacionados principalmente con variables de personalidad (editada bimensualmente desde 1962 por American Psychological Association, 1200 17th St. N. W.; Washington DC 20036).

Psychology of Women Quarterly. Publicada a partir de 1976 por la Division of the Psychology of Women de la American Psychological Association (Human Sciences Press, 72 Fifth Ave., New York, N. W. 10011). Está centrada fundamentalmente en estudios sobre la mujer.

Otras revistas interdisciplinarias que recogen bastantes artículos sobre el tema de los roles sexuales son:

Developmental Psychology: edición bimensual desde 1969 (American Psychological Association 1200 17th St. N. W.; Washington DC 20036).

Journal of Personality Assessment: edición bimensual desde 1970 (Society for Per sonality Assessment Inc.; 2814 SW Labbe Ave., Portland, DR 97221).

Journal of Clinical Psychology: edición cuatrimestral desde 1945 (Clinical Psychology Publishing Co. Inc., 4 Conant Square, Brandon, VT 05733).

Journal of Marriage and the Family: edición cuatrimestral desde 1939 (National Council on Family Relations, 1219 University Ave., SE Minneapolis, MN 55414).

Child Development: edición cuatrimestral desde 1930 (Society for Research in Child Development, Inc., University of Chicago Press, 5801 Ellis Ave., Chicago, IL 60637). 
Archives of Sexual Behavior: edición semestral desde 1971 ( Plenum Press, 233 Spring St., New York, NY 10013).

Perceptual and Motor Skills: edición bimensual a partir de 1949 (Ed. and Publ. C. H. Ammons and B. Ammons, Box 9229, Missoula, MT 59807).

Journal of Research in Personality: edición trimestral desde 1965 (Academic Press Inc. 111 Fifth Ave., New York, NY 10003).

Personality and Social Psychology Bulletin: edición cuatrimestral desde 1975 (Society for Personality and Social Psychology, Sage Publications, Inc., 275 S. Beverly Dr., Beverly Hills, CA 90212).

Journal of Psychology: edición bimensual desde 1936 (Journal Press Box 543, 2 Commercial St., Provincetown, MA 02657).

Journal of Homosexuality: edición cuatrimestral desde 1974 (Haworth Press, 28 E. 22nd St., New York, NY 10010).

Otras revistas pueden ser también consultadas para una mayor revisión del tema: Psychological Bulletin, Journal of Genetic Psychology, American Psychologist, Psychological Review, Developmental Psychologist, Human Development, Journal of Social Issues, Sings, International Journal of Women Studies, y sobre todo dos revistas australianas: Australian Psychologist y Australian Journal of Psychology.

Por último conviene informar de la aparición de algunos números monográficos sobre este tema en las revistas Archives of Sexual Behavior (1978, julio) y Child Development (1978, marzo), y de la presencia de varios artículos, entre seis y ocho, dedicados al tema en Professional Psychology (1981, febrero), Counseling Psychologist $(1979,8,1)$ y Journal of Social Issues (1978, Win.).

\section{COMPILACIONES}

Están recogidas en este apartado las obras (readings) editadas con posterioridad a 1974. Se remitirá al lector a su crítica en el Contemporary Psychology, siempre que sea posible.

Ashmore, D., y DeL Bocca, F. K. (eds.). The social psychology of female-male relations: A critical analisis of central concepts, Nueva York; Academic Press, 1986.

Caplan, P. (ed.). Psychology changing for women, Montreal: Eden Press Women's Publications, 1983.

CARney, C. G., y MC MAHON, S. L. (ed.). Exploring Contemporary Male-Female Roles: A facilitator's Guide, La Jolla, California: Inov. Associates, $1977{ }^{24}$.

CARTER, L. A, y SCOTT, A. F. (ed.). Women and men: changing roles, relationships and perceptions, Nueva York: Aspen Institute for Humanistic Studies, 1976.

KaPLAN, A. G. (ed.). Psychological androgyny, furtber considerations: a special issue of Psychology of women quarterly, Nueva York: Human Sciences Press, 1979.

KAPLAN, A. G., y BEAN, J. P. (eds.). Beyond sex-role stereotypes: readings toward a psychology of androgyny, Boston: Little Brown, 1976.

MAYO, C., y HENLEY, N. M. (eds.). Gender and nonverbal bebavior, Nueva York: Springer-Verlag, $1981^{25}$.

O'LEARY, V. E.; UNGER, R., y WALLSTON, B. S. (eds.). Women, gender and social psychology, Nueva Jersey: LEA, 1985.

ORTNER, J. B., y WHITEHEAD, H. (eds.). Sexual meanings, the cultural constructions of gender and sexuality, Nueva York: Cambridge University Press, 1981.

PARSONS, J. E. (ed.). The psychobiology of sex differences and sex roles, Washington: Hemisphere, Pub. Corp., $1980^{26}$.

PleCK, J. H., y SAWYeR, J. (eds.). Men and masculinity, Nueva Jersey: Prentice-Hall, 1974.

Reinisch, J. M.; Rosenblum, L. A., y SANDers, S. A.(eds.). Masculinity, femenity: Concepts and definitions, Nueva York: Oxford University Press (en prensa).

RICHARDSON, B. L., y WIRTENBERG, J. (eds.). Sex role research: Measuring social change, Nueva York: Praeger, $1983^{27}$. 
VETTERLING-BRAGGIN, M. (ed.). Feminity, masculinity and androgyny: a modern philosophical discussion, Totowa, Nueva Jersey: Rowman and Little-field, $1982^{28}$. WIDOM, C. S. (ed.). Sex roles and psychopatbology, Nueva York: Plenum Press, $1984^{29}$.

Por su especial relevancia hacemos mención de los siguientes capítulos incluidos en obras de compilación conocidas ampliamente:

Huston, A. C. «Sex typing», en: P. H. Mussen (ed.): Handbook of child psychology. Socialization, personality and social development (vol. IV), Nueva York: John Wiley and Sons, 1983.

SPENCE, J. T.; DeaUX, K., y HelmReich, R. L. «Sex roles in contemporary american society», en: G. Lindsey y E. Aronson (eds.). Handbook of Social Psychology, Reading Mass.: Addison-Wesley, 1985.

\section{ACTAS Y SERIES}

Se han celebrado reuniones científicas en torno al tema de los roles sexuales, identidad sexual, etc., y más específicamente de la androginia. A continuación reseñamos las dos más importantes:

Psychology and Gender. Nebraska Symposium on Motivation, 1984, vol. 32, Lincoln: University of Nebraska Press, 1985.

Symposium on conceptual and instrumental issues in Androgyny. Meeting of the American Psychological Association, Toronto, Canadá, agosto de 1978.

En cuanto a las series publicadas, aunque no corresponden fielmente a tal denominación, podemos señalar las ya clásicas que dedican varios artículos al tema:

MAHer, B. A., y MAHER, W. B. (eds.). Progress in experimental personality research, Nueva York: Academic Press, $1981^{30}$.

BERKOWITZ, L. (ed.). Advances in experimental social psychology. Vol. 14. New York: Academic Press, $1981^{31}$.

WHEELER, L. (ed.): Review of personality and social psychology: 1. Beverly Hills, California; Sage 1980.

WHEELER, L. (ed.): Review of personality and social psychology: 2. Beverly Hills, California; Sage, $1981^{32}$.

\section{PSYCHOLOGICAL ABSTRACTS (FUENTES SECUNDARIAS)}

Para la localización de trabajos sobre los roles sexuales en general y sobre el aspecto más concreto objeto de la presente guía, hemos utilizado los siguientes descriptores de Psychological Abstracts: Androgyny, Masculinity, Feminity, Sex roles y Sex roles attitudes. En cualquiera de ellos, pero muy especialmente en los tres primeros se encuentran los trabajos referidos al tema ${ }^{33}$.

En la Tabla 2 figura un ejemplo concreto cogido de uno de los índices semestrales del P. A. bajo los términos «feminity» y «androgyny». El lector puede observar la temática que subyace a ambos constructos.

\section{REVISIONES}

Mediante las revisiones elaboradas por otros autores, el investigador puede «hacerse», con un mínimo esfuerzo, con una panorámica y unas conclusiones generales sobre el tema que le interese. La utilización del Psychologi- 
Femininity

absence of denial of femininity \& underlying psychodynamics, anorexia nervosa, $28 \& 17$ yr old female clients, 26353.

achievement motivation, masculinity-femininity, college students, 31366

alcohol drinking attitudes \& patterns \& college adjustment, masculine \& feminine \& androgynous female college students, 29962

attitudes toward femininity, $14-31 \mathrm{yr}$ old anorexic females, 31664 changing views of femininity \& feminine identity \& reproduction, 6655

De Cecco-Shively Social Sex Role Inventory vs Bem Sex Role Inventory vs Personal Attributes Questionnaire, assessment of social sex role, heterosehomosexual males \& females, 19379

degreee of homosexuality \& gender identity, homosexual males, Australia vs Sweden vs Finland, 20415

development of feminine traits, female children, 20247

factor analysis, structure \& consistency of Personal Research Form ANDRO scale, college students, 16671

femininity \& disposition to maternity in treatment process, psychoanalysts, paper presented at 33rd International Psychoanalytic Congress, 1983, 31916

femininity vs masculinity vs androgyny \& sex differences, parent-infant interaction, $21-35$ yr old parents, 20327

GPA \& sex role orientation, attributions for academic achievement, androgynous vs feminine female college students, 28560

gried over death \& femininity as psychic process, 15064

heterosexuality vs homosexual preference \& fatherson interpersonal distance $\&$ childhood gender identity, pedophilic vs pedohebephilic vs gynephilic vs androphilic males, 20400

instructions \& rating scales of A. L. Edwards vs S. L. Bem, item selection for Masculinity \& Femininity scales of Bem Sex Role Inventory, college students, 22145

masculine instrumentality vs feminine expressivity, personality \& social adjustment, $18-25$ yr old female college students vs psychiatric patients, 17825

masculine vs feminine \& socially desirable vs undesirable personality characteristics, person evaluation, college students, 4039

masculinity \& femininity \& body cathexis \& self esteem, bisexual vs homosexual vs heterosexual females with average age of $25.2 \mathrm{yrs}, 20405$

masculinity \& femininity \& marital satisfaction \& response to behavioral marital therapy, distressed vs nondistressed husbands vs wives, 23835

masculinity-femininity \& sex, self esteem \& peer acceptance, Black vs White 14-18 yr olds, replication of C. M. Massad's study, 31122

masculinity vs femininity, assertiveness as measured by self report \& role playing, college students, 19435

motor behavior, 4-10 yr old feminine vs masculine males vs feminine females, 3776

narcissism \& femininity \& artistic creation, review of psychoanalytic work of L. Salome, 22055

relationships with parents \& sexual partners, transsexual vs effeminate vs nonefferminate homosexual males, 20417

roles \& status, mascuiine vs feminine adolescents, $\mathrm{Tu}$ nisia 6472 self esteem \& body image \& sexual satisfaction, androgynous vs masculine vs feminine vs undifferentiated unmarried female college students, 4023

self monitoring, expressive masculinity-femininity, college students, 12269

self reported social desirability, feminine vs masculine vs androgynous vs undifferentiated college students, 20512

sex \& masculinity vs femininity, hemispheric orientation, adults, 622

sex role identification, male vs female sexual domints vs submissives, 3921

sex role orientations, male alcoholic vs nonalcoholic veterans vs male college students, 18018

sex role stereotypes \& gender identity \& perceptions of parents, heterosexual vs homosexual males, 20406

social sex roles \& gender identity, homosexuals, 20414 socially desirable $\&$ undesirable masculine $\&$ feminine traits \& body weight \& body image \& dieting concern, college students, 6647

stereotypes of male \& female homosexuals \& sex role attitudes, $17-64$ yr olds, 20420

\section{Androgyny}

alcohol drinking attitudes \& patterns \& college adjustment, masculine \& femenine \& androgynous female college students, 29962

androgyny \& management styles, business managers, 24666

androgyny vs sex typing, beliefs in gender polarity, male vs female college students, 1278

clients' masculine vs feminine vs androgynous sex roles, clinical judgment, social workers, 4966

comparative factor analysis of Personal Attributes Questionnaire \& Bem Sex Role Inventory, measurement of androgyny, 17-45 yr olds, 5595

continuous metric for correlational analyses, measurement of androgyny, 16793

GPA \& sex role orientation, attributions for academic achievement, androgynous vs feminine female college students, 28560

psychological androgyny \& self image, adaptability \& coping style, college students, 1275

scoring androgyny as continuous variable, reply to S. Blackman's criticism, 12104

scoring systems for androgyny as sum of femininity \& masculinity, regression analysis, 16662

self esteem \& body image \& sexual satisfaction, androgynous vs masculine vs feminine vs undifferentiated unmarried female college students, 4023

self reported social desirability, feminine vs masculine vs androgynous vs undifferentiated college students, 20512

sex role orientation, loneliness in adolescence, female vs male junior \& senior high school students, 1042

sex roles \& androgyny \& group size, helping choking victim, college students, 12171

Se' physical attractiveness \& androgyny \& Os' sex \& sex roles, social perceptions, college students, 12201

tolerance for ambiguity, ability to read facial expressions, androgynous vs nonandrogynous college students, 28643 
cal Abstracts y del Annual Review of Psychology pueden proporcionar una información bastante valiosa y completa en este punto.

Merecen la pena para destacarse las siguientes:

BASSOFF, E. S.; GLASS, G. V. «The relationship berween sex roles and mental health: A metaanalysis of twenty-six studies». Counseling Psychologist, 1982, 10, 105-112.

CONSTANTINOPLE, A. «Masculinity-feminity: An exception to a famous dictum? Psychological Bulletin, 1973, 80, 389-407.

DEAUX, K. «Dex and gender». Annual Review of Psychology, 1985, 36, 49-81.

JOHNSON, M. M. «Fathers and "feminity" in daughters. A review of the research». Sociology and Social Research, 1982, 67, 1-17.

KELLY, J. A., y WORRELL, J. «New formulations of sex roles and androgyny: a critical review». Journal of Consulting and Clinical Psychology, 1977, 45, 1101-15.

NATHANSON, C. A. «Illness and the feminine role: A theoretical review». Social Science and Medicine, 1975, 9, 57-62.

PYKE, S. W., y GRAHAM, J. M. «Gender schema theory and androgyny: A critique and claboration\%. International Journal of Women's studies, 1983, 6, 3-17.

RoBINSON, B. E., y GREEN, M. G. «Beyond androgyny: The emergence of sex-role transcendence as a theoretical construct». Developmental Review, 1981, 1, 247-265.

TAYLOR, M. C., y HALL, J. A. «Psychological androgyny: Theories, methods, and conclusions». Psychological Bulletin, 1982, 92, 347-366.

Una excelente revisión desde una perspectiva evolutiva constituye el trabajo de Shepherd-Look:

SHEPHER-LOOK, D. L. «Sex differentiation and the development of sex roles, en B. P. Wollman (ed.): Handbook of developmental psychology, Nueva Jersey: Prentice Hall, 1982.

\section{CATALOGOS}

Citaremos a continuación los principales trabajos bibliográficos a los que puede acudir el investigador interesado en alguno de los siguientes aspectos:

Astin, H. S., y PASelman, A.; Fisher, A. Sex roles: $A$ research bibliograpby. Rockviile, Md: National Institute of Mental Health, Washington, 1975.

BAZIN, N. T. «The concept of androgyny. A working bibliography». Women's studies, 1974, 2, 217-235.

GRADY, K. E.; BRANHON, R.; PLECK, J. H. The male sex role: a selected and annotated bibliography. Rockville, Md: National Institute Of Mental Health; Washiington, 1979.

WHITLEY, B. E. «Sex role orientation and mental health: an annotated research bibliography». Catalog of selected Documents in Psychology, 1980, 10, 90.

\section{CONCLUSIONES}

Aunque a este archivo bibliográfico le faltan referencias de temas muy cercanos y colindantes con el de la masculinidad y feminidad (referencias socioantropológicas), creemos que puede servir de base para, en un futuro, llegar a coleccionar las obras más valiosas de esta extensísima área de estudio.

En este sentido, los autores están interesados en realizar otras guías con respecto a temas como la psicología de la mujer y del varón y otros temas anexos. 
1 Singer, J. I. Androgyny. Toward a new theory of sexuality, Londres: Routledge and Kegan Paul, 1977.

2 BEM, S. L. Bem Sex-Role Inventory: Professional Manual, Palo Alto, California: Consulting Psychologist Press, 1981.

3 Véase Vontemporary Psychology, 1984, 11, 765.

4 Véase Contemporary Psychology, 1984, 4, 355.

s Véase Vontemporary Psychology, 1981, 1, 39.

${ }^{6}$ Desde un punto de vista más cotidiano del tema pueden consultarse:

Pérez, C. D. Masculino-Femenino o la bella diferencia, Buenos Aires: Paidos, 1982.

VIlLARREAL, J. Similitudes y contrastes psicológicos entre lo masculino y lo femenino, Buenos Aires, 1972.

7 Aunque hemos tratado de no meternos en el tema de los estereotipos sexuales, podemos señalar los siguientes trabajos realizados con muestras españolas que pueden ser de interés.

Barberá, E.; Martinez Benlloch, I., y PAStor, R. «Estereotipos sexuales infantiles y empleo materno: una réplica del estudio de Jones y McBride». Psicológica, 1983, 1, 143-151.

BarberA, E.; Martínez Benlloch, I., y Pastor, R. «La escuela entre la uniformidad y la diferencia: un estudio sobre la representación del estereotipo sexual». Psicológica, 1984, 5, 1, 101-113.

MUSiro OCHOA, G. «El estereotipo masculino y femenino en el contexto socio-cultural valenciano». Psicológica, $1980,1,129-142$.

${ }^{8}$ Jiménez-Fernf́ndeZ, A, y cols. «Guía documental del estrés». Estudios de Psicología, 1983, 13, 111-121.

9 Thesaurus of Psychological Index, 3.a ed., Washington D. C., APA, 1982.

10 Véase Contemporary Psychology, 1981, 9, 698.

11 Véase Contemporary Psychology, 1982, 7, 560.

12 Véase Contemporary Psychology, 1979, 24, 5.

${ }_{13}$ Constituye, a nuestro entender, el mejor libro sobre el tema.

14 Véase Contemporary Psychology, 1978, 23, 11.

15 Véase Contemporary Psychology, 1982, 4, 271.

16 Véase Contemporary Psychology, 1980, $25,7$.

17 Véase Contemporary Psychology, 1982, 8, 606.

18 Ofrece una panorámica del tema de los roles sexuales desde la biología, psicoanálisis, sociología, psicología y antropología.

19 Véase Contemporary Psychology, 1982, 4, 289.

Es una ampliación de su libro Sex role issues in metal bealth, 1980.

20 Véase Contemporary Psychology, 1977, 22, 12.

21 Véase Contemporary Psychology, 1979, $24,1$.

${ }^{22}$ Véase Contemporary Psychology, 1981, 5, 380.

23 Véase Contemporary Psychology, 1978, 23, 3.

${ }^{24}$ Esta obra no satisface las expectativas creadas por un título tan prometedor.

25 Véase Contemporary Psychology, 1983, 2, 128.

26 Véase Contemporary Psychology, 1980, 25, 10.

${ }^{27}$ Véase Contemporary Psychology, 1984, 4, 336.

28 Véase Contemporary Psychology, 1983, 1, 70.

29 Véase Contemporary Psychology, 1984, 8, 682.

30 Véase Contemporary Psychology, 1982, 4, 237.

31 Véase Contemporary Psychology, 1982, 5, 405.

${ }^{32}$ Véase Contemporary Psychology, 1982, 4, 277.

${ }^{33}$ Para una descripción de su utilización véase:

ORTEGA, J. E., y FERNANDEZ-DOLS, J. M. Fuentes documentales en Psicología, Madrid: Debate, 1980. 